\title{
J'imagine comme verbe et marqueur pragma- tique : une analyse diachronique
}

\author{
Stefan Schneider ${ }^{1,1}$ \\ ${ }^{1}$ Institut für Romanistik, Karl-Franzens-Universität Graz, Merangasse 70, 8010 Graz, Autriche
}

\begin{abstract}
Résumé. La présente étude aborde la pragmatique du marqueur déverbal j'imagine en appliquant les concepts de la grammaire de construction diachronique (voir Hilpert 2013 ; Traugott \& Trousdale 2013 ; Barðdal \& Gildea 2015 ; Lowrey 2018 : 152-154). Plus précisément, elle examine les occurrences à la première personne du singulier du présent de l'indicatif du verbe cognitif français imaginer dans la base Frantext. La question qui se pose est de comprendre comment et quand j'imagine est devenu un marqueur déverbal pendant son évolution à partir du moyen français jusqu'au français moderne. Pour observer l'émergence de la construction de marqueur, premièrement, on établit l'ensemble des constructions dans lesquelles apparait j'imagine (= son réseau de constructions) et, dans une deuxième phase, on étudie son évolution chronologique.
\end{abstract}

\begin{abstract}
The present study describes the pragmatics of the deverbal marker j'imagine by applying the concepts of diachronic construction grammar (see Hilpert 2013; Traugott \& Trousdale 2013; Barðdal \& Gildea 2015; Lowrey 2018: 152-154). More precisely, first person singular present indicative instances of the French cognitive verb imaginer are examined in a corpus, namely the Frantext database, to find how and when j'imagine developed into a deverbal marker in the course of its evolution from Middle to Modern French. To trace the emergence of the marker construction, we first establish the set of the constructions in which j'imagine occurs (= its constructional network) and, in a second step, examine its chronological development.
\end{abstract}

\section{Introduction}

La présente étude aborde la pragmatique des marqueurs de discours avec les concepts et outils de la grammaire de construction diachronique (voir Hilpert 2013; Traugott \& Trousdale 2013 ; Barðdal \& Gildea 2015 ; Lowrey 2018 : 152-154). Elle analyse le verbe cognitif français j'imagine dans les textes de la base Frantext et examine son évolution à partir du moyen français jusqu'au français moderne.

À côté de l'usage comme verbe «plein » avec le signifié conceptuel cognitif 'concevoir l'image d'un être ou d'une chose (sous tel ou tel aspect, dans telle ou telle circonstance)',

\footnotetext{
${ }^{1}$ stefan.schneider@uni-graz.at
} 
j'imagine peut apparaître en position détachée et isolée et y assumer le signifié procédural 'croire, penser, supposer', c'est-à-dire qu'il guide l'interprétation et l'évaluation épistémique des éléments linguistiques qui se trouvent dans sa portée :

(1) parce que je le vis sousrire deux ou trois fois, jugeant, comme je m'imagine, l'impatience où j'estois, par luy-mesme. (Honoré d'Urfé, L'Astrée : $t$. 4 : 4ème partie : livres 1 à 12, 1627)

(2) / Vous l'avez fait, je m'imagine, / ce petit rondeau qui rafine / tous les rondeaux de ce temps-cy. / (Claude Malleville, Euvres poétiques, 1649)

(3) la variété des objets et l'abondance des idées qui n'ont jamais, j'imagine, passé par aucune tête que la mienne. (Denis Diderot, Lettres à Sophie Volland: t. 2 (26 sept. 1762-1774), 1774)

Le verbe en position détachée et isolée avec un signifié procédural est appelé marqueur déverbal. L'examen se concentre sur la première personne du singulier du présent de l'indicatif parce que c'est la forme qui montre la tendance la plus forte à développer la fonction de marqueur.

L'article est structuré de la manière suivante : la deuxième section fait le point sur les études précédentes; la troisième section explique les questions posées et les données sur lesquelles s'appuie la présente étude; la quatrième section fournit une description des constructions dans lesquelles apparaît j'imagine; la section suivante examine les premières occurrences de chaque construction et l'évolution des réseaux de constructions et la section finale présente les conclusions.

\section{2 Études précédentes}

Les verbes détachés et isolés avec un signifié procédural existaient déjà en latin écrit (voir Pinkster 1988 [1984]) : 308-309) et, comme on peut le supposer, aussi en latin parlé. De fait, le latin possédait un répertoire assez riche de ces marqueurs.

Le volume de Hofmann (1951) sur le latin familier et populaire contient de nombreux exemples de ces verbes. Certains dérivent des impératifs (voir Hofmann 1951 : 37-39, 126127). En ce qui concerne les verbes cognitifs, Hofmann (1951 : 106-107) mentionne censeo, credo, opinor et puto. Le verbe illocutoire inquam, surtout employé dans les actes de langage assertifs, et les verbes illocutoires obsecro, oro, quaeso et rogo, employés dans les invocations, questions et requêtes, présentent un comportement similaire (voir Hofmann $1951: 125-131)$ :

(4) per mihi, per, inquam, gratum feceris (Cicéron, Epistulae ad Atticum, 1, 20, 7)

'Tu me feras, je dis, une faveur.'

(5) dic obsecro hercle serio (Plaute, Asinaria, 29)

'Dis-moi, je te prie, par Hercule, sincèrement, ce que je te demande.'

(6) tam casta est, rogo, Thais ? (Martial, 4, 84, 4)

'Thais est-elle, je demande, tellement pudique ?'

Hofmann (1951 : 127) note aussi que la première personne du singulier du futur de l'indicatif du verbe amare peut apparaître dans les requêtes comme marqueur avec le signifié de 's'il vous/te plaît' (voir aussi Charnavel 2007 : 160; Holford-Strevens 2010 : 334-336). Certains marqueurs sont introduits par la conjonction si. Celle-ci peut se fondre avec le verbe, ce qui produit un nouveau mot (univerbation). Par exemple, si vis apparaît aussi comme sis, si audes comme sodes (voir Hofmann 1951: 132-134). À maintes reprises, Hofmann (1951 : 37, 106, 126-127) souligne que ces verbes se comportent comme des particules invariables. 
Les études d'Albrecht (1964) sur la parenthèse dans Ovide, et de Risselada (1989) sur les parenthétiques illocutoires dans les actes de langage directifs apportent d'autres preuves. Bolkestein (1998a, 1998b) analyse les verba sentiendi parenthétiques du latin, surtout dans les lettres de Cicéron. Les verbes qui apparaissent dans les lettres comme « modalization in one way or another of the degree of strength with which the speaker posits his utterance " (Bolkestein 1998b: 11) sont arbitror, censeo, credo, existimo, opinor, puto et sentio. Bolkestein (1998a : 23) affirme que ces verbes se comportent d'une manière « quite similar to the way in which other modalising expressions function, such as sentence adverbs and particles ». Bolkestein (1998a : 30, 1998b : 4, 11) cite entre autres les exemples suivants pour credo, opinor et puto :

(7) Nam Quintus frater, homo mirus, qui me tam ualde amat, omnia mittit spei plena, metuens, credo, defectionem animi mei (Cicéron, Epistulae ad Atticum, 3, 18, 2)

'Mon frère Quintus, cet homme admirable, qui m'aime si tendrement, ne m'écrit que des lettres pleines d'espérance. Il craint, je crois, que je ne tombe dans le découragement.'

(8) Melitam igitur, opinor, capessamus, dum quid in Hispania (Cicéron, Epistulae ad Atticum, 10, 9, 1)

'Alors, partons pour Malte, je dirais, allons-y attendre les événements d'Espagne.'

(9) Cuius modi velim, puto, quaeris (Cicéron, Epistulae ad familiares, 8, 3, 3)

'Tu me demanderas, je pense, quelle sorte de chose je veux.'

Les verbes existimo, opinor et puto peuvent être introduits par la conjonction ut (Bolkestein 1998a : 27, 1998b : 13), qui, dans ce contexte, signifie 'comme' ou 'selon'. Les verbes arbitror et sentio sont toujours introduits par $u t$, tandis que credo n'apparaît jamais avec cette conjonction. Häusler (2000), Charnavel (2007), Reinhardt (2010 : 224-225), Coleman (2010 : 292), Kay (2010 : 322-323) et Ghezzi \& Molinelli (2014) donnent d'autres exemples de marqueurs déverbaux latins.

La Base de français médiéval (BFM), en plus des textes de l'ancien français, donne la possibilité d'analyser la partie latine du corpus Le passage du latin au français (PaLaFra). Ce corpus comprend 187 textes latins de la période mérovingienne, qui datent du $5^{\mathrm{e}}$ jusqu'au $10^{\mathrm{e}}$ siècle. Ces textes (vies de saints, chroniques, chartes, recueils de lois, lettres et formulaires) contiennent de nombreux exemples de verbes détachés et isolés avec un signifié procédural :

(10) de magno, credo, semine descendit (Auspicus, Epistula Auspici episcopi ecclesiae Tullensis ad Arbogastem comitem Treferorum, env. 460)

'[il] descend, je crois, d'un grand lignage'

(11) iam enim, ut spero, etsi sensu rustico prolixum produximus sermonem (Audoinus, Vita Eligii episcopi Noviomagensis, 670/680)

'mais déjà, comme j'espère, nous faisions un discours prolixe avec pourtant un signifié simple'

Par conséquent, on peut affirmer que les auteurs du latin classique écrit avaient à leur disposition un modèle constructif pour maîtriser l'usage détaché et isolé de certains verbes. Cette connaissance a été transmise aux auteurs du latin tardif et, comme on le verra cidessous, aussi aux auteurs des langues romanes médiévales.

Les travaux de Féron (2005), Glikman (2012), Schneider (2012, 2013), Haßler (2014), Schneider \& Glikman (2015) et Glikman \& Schneider (2018) abordent la question de la présence des marqueurs déverbaux cognitifs en ancien et moyen français et dans les langues romanes médiévales en général. On peut résumer ces études en disant que des expressions verbales détachées et isolées basées sur les verbes croire, cuidier, espérer, 
penser et sembler existent dès les premiers textes (cf. aussi Buridant 2019 : 1109). Même si leurs verbes de base n'ont pas un signifié conceptuel cognitif, comme dans le cas de sembler, elles assument un signifié procédural épistémique.

Il n'existe aucune étude sur l'usage détaché et isolé de j'imagine, mais une étude de Schneider (2018) examine son correspondant espagnol et analyse l'évolution de quatre verbes espagnols : creo apparaît en position détachée et isolée à partir du $13^{\mathrm{e}}$ siècle, pienso à partir du $15^{\mathrm{e}}$, imagino à partir du $16^{\mathrm{e}}$ et supongo dès le $17^{\mathrm{e}}$. Avant que ces verbes puissent être employés comme marqueurs déverbaux détachés et isolés, ils doivent passer par deux étapes préalables : premièrement, ils doivent acquérir la capacité d'introduire et de régir des clauses et, deuxièmement, ils doivent acquérir un signifié procédural épistémique.

\section{Questions et données}

En résumé, en latin et dans les langues romanes médiévales, nous avons affaire à trois groupes de marqueurs déverbaux :

1. plusieurs d'entre eux sont présents en latin, mais disparaissent plus tard pendant la transition vers les langues romanes ;

2. d'autres marqueurs sont présents en latin et se sont maintenus dans les langues romanes ;

3. nombre de marqueurs déverbaux romans dérivent des verbes ou, plus généralement, des lexèmes présents en latin, mais ils n'apparaissent pas en position détachée et isolée dans cette langue : leur fonction de marqueur est nouvelle.

Le verbe français j'imagine fait partie de ce dernier groupe. Il se base sur les verbes latins imaginor et imagino, qui avaient un signifié conceptuel cognitif à côté de leurs autres signifiés, mais qui ne sont pas documentés en position détachée et isolée.

Grâce aux marqueurs du deuxième groupe, les auteurs, et probablement aussi les locuteurs, avaient conscience de cette construction spécifique. La présence des marqueurs basés sur des verbes du troisième groupe, comme com je cuit, ce cuit et je cuit (du lat. cogito), (ce) me semble et com il me semble (du lat. tardif similo), dans les textes français du $12^{\mathrm{e}}$ siècle (voir Schneider \& Glikman $2015: 174,176$ ) prouve que très tôt les auteurs ont su adapter à ce modèle des verbes qui précédemment n'étaient pas employés de cette manière. La question qui se pose donc est de comprendre comment j'imagine est entré dans cette construction.

Pour y répondre, il faut savoir dans quelles constructions apparaît j'imagine et comment elles se développent chronologiquement. Afin d'observer l'émergence de la construction détachée et isolée parmi les autres, il faut identifier toutes les constructions dans lesquelles apparaît j'imagine pendant l'entière période chronologique examinée. Il faut donc établir l'ensemble des constructions dans lesquelles apparaît j'imagine ou son réseau de constructions général. Celui-ci change au fil du temps, c'est-à-dire que l'usage de certaines constructions peut s'intensifier ou diminuer, ou bien encore que des constructions peuvent disparaître complètement du réseau. C'est la raison pour laquelle, dans un deuxième temps, on peut établir des réseaux de constructions spécifiques pour des périodes chronologiques successives.

L'analyse s'appuie sur deux types de données. Dans un premier temps, les trois premières occurrences de chaque construction ont été relevées. Chacune des premières occurrences provient d'un auteur différent. Autrement dit, plusieurs occurrences dans un même document ou chez un même auteur ont été écartées. Cette partie de la recherche examine tous les textes de la base Frantext dans leur intégralité. Les premières occurrences montrent à quel moment une construction spécifique commence à apparaître et permettent d'établir une séquence chronologique approximative. $\mathrm{Vu}$ que les premières apparitions ne nous apprennent rien sur la diffusion, la raréfaction ou la disparition d'une construction 
dans la période suivante, dans un deuxième temps, ces données ont été complétées et intégrées au moyen d'une analyse de quatre réseaux de constructions spécifiques établis à partir de quatre périodes chronologiques successives. Afin de garantir une comparabilité optimale à travers les genres de texte différents, ces réseaux spécifiques ont été constitués exclusivement à partir des occurrences tirées des passages au discours direct.

Ces derniers présentent deux avantages: dans une certaine mesure, ils sont diachroniquement stables et contiennent en outre plus de traces d'oralité que d'autres passages textuels. Même si leur présence varie considérablement, on les trouve dans quasiment tous les textes et, surtout, pendant toute l'histoire du français, dès les premières vies de saints jusqu'aux romans des $18^{\mathrm{e}}, 19^{\mathrm{e}}$ et $20^{\mathrm{e}}$ siècles, en passant par les chansons de geste, les chroniques et le théâtre classique. Les genres textuels varient à de nombreux égards au cours de l'histoire du français, mais les passages au discours direct restent stables et gardent leurs propriétés fondamentales. On peut donc présumer qu'ils garantissent une base empirique plus fiable.

Un maximum de dix occurrences par auteur a été choisi. De plus, les longs monologues présents dans quelques textes ont été exclus. Évidemment, la présence et la fréquence du discours direct varient considérablement selon les textes de Frantext. C'est la raison pour laquelle les quatre tranches chronologiques à la base des quatre réseaux de constructions spécifiques ne peuvent pas avoir la même durée. Pour réunir une soixantaine d'occurrences de j'imagine par réseau, on a été obligé d'analyser des quantités de texte différentes. Chaque réseau se base donc sur un ensemble de textes d'une tranche chronologique différente : 1610-1682 pour le premier réseau, 1723-1738 pour le deuxième, 1821-1857 pour le troisième et 1920-1935 pour le quatrième. Une soixantaine d'occurrences par réseau spécifique représente certes une quantité relativement restreinte, mais il s'agit de montrer les potentialités de l'approche de la grammaire de construction diachronique plutôt que de fournir des données empiriques exhaustives.

\section{Constructions et sous-constructions}

\subsection{Introduction}

L'analyse révèle que, pendant la période historique examinée, j'imagine apparaît dans de nombreuses constructions différentes. En suivant l'approche de la grammaire de construction diachronique (voir Hilpert 2013; Traugott \& Trousdale 2013; Barðdal \& Gildea 2015; Lowrey 2018: 152-154), nous décrivons les constructions à plusieurs niveaux d'abstraction, en l'occurrence à deux niveaux. À un niveau élevé d'abstraction et de schématisation, nous avons identifié les constructions suivantes :

- verbe régissant une expression nominale : $[\mathrm{V} \Rightarrow \mathrm{EN}]$;

- verbe régissant un infinitif : $[\mathrm{V} \Rightarrow \mathrm{INF}]$;

- verbe régissant une clause : $[\mathrm{V} \Rightarrow \mathrm{C}]$;

- phrase contenant une expression verbale détachée : [P $\rightarrow . \rightarrow E V D]$.

Les prochaines sous-sections donnent une description des constructions et sousconstructions respectives.

\subsection{Verbe régissant une expression nominale}

Dans la construction $[\mathrm{V} \Rightarrow \mathrm{EN}]$, le verbe apparaît avec un ou plusieurs arguments de caractère nominal. Les arguments revêtent des formes différentes. Ils peuvent être des pronoms clitiques ou des syntagmes nominaux, mais aucun argument n'est une clause, c'est- 
à-dire que, sémantiquement, les arguments sont des termes et pas des propositions. En fonction de la forme de l'expression nominale, et sans prendre en compte les éléments attributifs, on peut distinguer deux sous-constructions :

(12) Le plus contemplatif et prudent homme, quand je l'imagine en cette assiette [...] (Michel de Montaigne, Essais : t. 2 (livre 3), 1592)

(13) J'imagine infinies natures plus hautes et plus reglées que la mienne; (Michel de Montaigne, Essais : t. 2 (livre 3), 1592)

Dans la première construction, le verbe régit un pronom clitique direct, dans la deuxième, il régit un syntagme nominal.

\subsection{Verbe régissant un infinitif}

Le verbe, éventuellement accompagné d'un pronom clitique indirect, régit un infinitif directement ou moyennant une préposition :

(14) / que tous mes sentimens soient meslez d'une rage, / qu'au lict je m'imagine estre dans un naufrage, / tomber d'un precipice et voir mille serpens / dans un cachot obscur autour de moy rempans. / (Théophile de Viau, Euvres poétiques : 2e partie, 1623)

(15) J'imagine desja de vous voir bras / à brastascher d'une secousse à vous jetter à bas, / (Antoine de Montchrestien, Les Lacènes ou la constance, 1601)

\subsection{Verbe régissant une clause}

Dans la construction $[\mathrm{V} \Rightarrow \mathrm{C}]$, un argument du verbe est représenté par une clause, c'est-àdire par une phrase régie :

(16) / J'ymagine que tes cuers m'aint / Et que li miens en toy remaint, / (Guillaume de Machaut, La fonteinne amoureuse, 1361)

Ici, le verbe imagine possède un signifié conceptuel cognitif qu'on peut paraphraser avec 'concevoir l'image d'un être ou d'une chose'. Le verbe est au premier plan et représente l'élément de base de l'énoncé entier. La phrase J'ymagine que tes cuers m'aint décrit une impression de l'auteur. On peut résumer sa structure par [je [V=ASS $\Rightarrow[q u e=\mathrm{C}]]]$.

La construction, tout en restant formellement identique, peut cependant présenter une structure sémantique et pragmatique assez différente :

(17) / Qui les meut [les fillettes] a ce ? G'ymagine, / Sans l'onneur des dames blasmer, / Que c'est nature femeninne / Qui tout unyement veult amer. / (François Villon, Le Testament, 1461)

Dans cet exemple, le verbe imagine a perdu son signifié conceptuel cognitif au profit d'un signifié général et abstrait proche de celui des marqueurs et qu'on peut définir comme procédural, épistémique et subjectif. Il n'est pas question de l'impression de l'auteur. C'est la clause qui est au premier plan. L'énoncé entier décrit l'état de choses 'C'est la nature féminine qui tout uniment veut aimer'. Imagine ne représente que la prise en charge réduite de l'auteur. On peut résumer cette structure par [je $[\mathrm{V} \Rightarrow[q u e \Rightarrow \mathrm{C}=\mathrm{ASS}]]]$. Il y a donc un décalage entre la forme syntaxique d'un côté et la structure sémantique et pragmatique de l'autre. On trouve ce décalage soit dans les phrases avec une clause finie soit dans celles avec une clause infinitive.

\subsection{Phrase avec une expression verbale détachée}


La construction [P ...,EVD] se compose d'une phrase (complète ou incomplète) qui contient une expression verbale détachée et isolée. On peut distinguer plusieurs sous-constructions.

Premièrement, on trouve la sous-construction dans laquelle l'expression détachée ne comprend que le verbe et son sujet clitique, comme on peut le voir dans l'exemple (3), repris ci-dessous :

(3) la variété des objets et l'abondance des idées qui n'ont jamais, j'imagine, passé par aucune tête que la mienne. (Denis Diderot, Lettres à Sophie Volland: t. 2 (26 sept. 1762-1774), 1774)

On peut la formaliser par $[\mathrm{P} \cdots,[j e \mathrm{~V}]+\cdots \mathrm{P}]$. C'est la structure qui domine dans les textes modernes et dans la langue parlée actuelle.

Dans un autre type de sous-construction, le verbe détaché et isolé est précédé d'un pronom objet indirect ou direct clitique. C'est le cas de l'exemple (2), repris ci-dessous :

(2) / Vous l'avez fait, je m'imagine, / ce petit rondeau qui rafine/tous les rondeaux de ce temps-cy. / (Claude Malleville, Euvres poétiques, 1649)

On peut formaliser la sous-construction dans l'exemple ci-dessus par la formule $[\mathrm{P} \rightarrow[j][\mathrm{PRO}=\mathrm{V}]]+\cdots \mathrm{P}]$.

Parfois, l'expression verbale détachée et isolée, normalement précédée d'un pronom objet clitique, est introduite par la conjonction comme, comme on peut le voir dans l'exemple (1), repris ci-dessous :

(1) parce que je le vis sousrire deux ou trois fois, jugeant, comme je m'imagine, l'impatience où j'estois, par luy-mesme. (Honoré d'Urfé, L'Astrée : $t .4$ : 4ème partie: livres 1 à 12, 1627) $\mathrm{P}]$.

On peut formaliser cette construction par la formule $[\mathrm{P} \cdots[$ comme $\Rightarrow[j e[\mathrm{PRO}=\mathrm{V}]]]+\cdots$

\section{Premières occurrences et réseaux de constructions}

Voyons maintenant l'évolution chronologique des constructions et sous-constructions. Tout d'abord, pour observer leur émergence, surtout celle de la construction détachée et isolée, nous examinons les trois premières occurrences de chacune d'entre elles. Cette partie de la recherche s'appuie sur tous les textes de la base Frantext dans leur intégralité.

Le diagramme 1 nous montre que la sous-construction $[j e[\mathrm{~V}=\mathrm{ASS} \Rightarrow[q u e \Rightarrow \mathrm{C}]]]$ est la première à apparaître, suivie un siècle plus tard par sa variante $[j e[\mathrm{~V} \Rightarrow[$ que $\Rightarrow \mathrm{C}=\mathrm{ASS}]]$, dans laquelle la clause est au premier plan.

Diagramme 1 : Les trois premières occurrences de j'imagine dans ses constructions et sousconstructions respectives

On note pourtant une différence de fréquence entre les deux sous-constructions. Les trois premières occurrences de $[j e[\mathrm{~V}=\mathrm{ASS} \Rightarrow[q u e \Rightarrow \mathrm{C}]]]$ s'étendent sur deux siècles. Autrement dit, il faut chercher deux siècles pour les trouver. Par contre, les trois premières occurrences 
de $[j e[\mathrm{~V} \Rightarrow[q u e \Rightarrow \mathrm{C}=\mathrm{ASS}]]]$ se concentrent seulement sur une cinquantaine d'années. Cette différence de fréquence sera encore plus évidente quand on examinera les quatre réseaux de constructions spécifiques. Même si, dans l'exemple (16), j'imagine régit une clause au subjonctif, l'emploi du subjonctif reste, en général, un phénomène isolé, limité surtout aux clauses après la négation.

$\mathrm{Au} 16^{\mathrm{e}}$ siècle, on voit apparaître la construction dans laquelle le verbe régit une expression nominale facultative $[\mathrm{V} \Rightarrow \mathrm{EN}]$. La construction avec l'infinitif fait son apparition à cheval entre le $16^{\mathrm{e}}$ et le $17^{\mathrm{e}}$ siècle. Pendant la même période, on voit apparaître la sous-construction détachée avec comme, la première de ce type. Quant à cette sousconstruction, il faut souligner que ses premières occurrences contiennent toujours des pronoms objet à côté du sujet (par exemple, comme je l'imagine, comme je m'imagine, comme je me l'imagine). Elle est chronologiquement suivie de la sous-construction dans laquelle le verbe détaché et isolé est précédé d'un pronom objet indirect clitique. La sousconstruction détachée $[\mathrm{P} \rightarrow[j e \mathrm{~V}] \ll \cdots \mathrm{P}]$ est chronologiquement la dernière et n'apparaît que pendant la deuxième moitié du $18 \mathrm{e}$ siècle. Mais son émergence est assez soudaine, vu que ses trois premières occurrences se concentrent sur une seule décennie.

Dans ce qui suit, nous complétons et intégrons l'analyse des premières occurrences au moyen d'une analyse de quatre réseaux de constructions spécifiques. Afin de garantir une comparabilité optimale à travers les différents genres de texte, les quatre réseaux de constructions ont été établis exclusivement à partir des occurrences tirées des passages au discours direct. Chaque réseau se base sur un ensemble de textes qui proviennent d'une tranche chronologique différente. Le tableau 1 affiche le nombre total d'occurrences et les pourcentages des constructions et sous-constructions de j'imagine dans les tranches 1610$1682,1723-1738,1821-1857$ et 1920-1935.

Tableau 1: Les pourcentages des constructions et sous-constructions de j'imagine

\begin{tabular}{|c|c|c|c|c|}
\hline Tranches & $1610-1682$ & $1723-1738$ & $1821-1857$ & $1920-1935$ \\
\hline Nombre d'occurrences & 57 & 58 & 66 & 62 \\
\hline$[\mathrm{V} \Rightarrow \mathrm{EN}]$ & $37 \%$ & $33 \%$ & $4 \%$ & $32 \%$ \\
\hline$[\mathbf{V} \Rightarrow \mathbf{I N F}]$ & $0 \%$ & $7 \%$ & $2 \%$ & $2 \%$ \\
\hline \multicolumn{5}{|l|}{$[\mathrm{V} \Rightarrow \mathrm{C}]$} \\
\hline$[j e[\mathrm{~V}=\mathrm{ASS} \Rightarrow[q u e \Rightarrow \mathrm{C}]]]$ & $10 \%$ & $9 \%$ & $3 \%$ & $5 \%$ \\
\hline$[j e[\mathrm{~V} \Rightarrow[q u e \Rightarrow \mathrm{C}=\mathrm{ASS}]]]$ & $51 \%$ & $48 \%$ & $53 \%$ & $24 \%$ \\
\hline \multicolumn{5}{|l|}{ 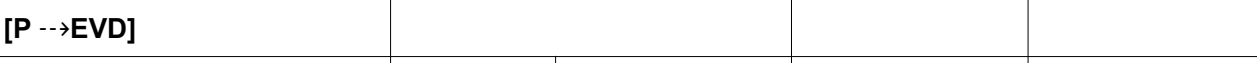 } \\
\hline$[\mathrm{P} \rightarrow \rightarrow[j e \mathrm{~V}] \stackrel{\cdots}{ }]$ & $0 \%$ & $0 \%$ & $27 \%$ & $37 \%$ \\
\hline$[\mathrm{P} \cdots[[j e[\mathrm{PRO} \Leftarrow \mathrm{V}]] \stackrel{\cdots P}{\mathrm{P}]}$ & $0 \%$ & $0 \%$ & $6 \%$ & $0 \%$ \\
\hline$\left[\mathrm{P} \rightarrow \rightarrow[\text { comme } \Leftarrow[j e \mathrm{~V}]]^{\leftrightarrow \cdots \mathrm{P}}\right]$ & $2 \%$ & $3 \%$ & $5 \%$ & $0 \%$ \\
\hline
\end{tabular}

Même si, dans le diagramme 1 , j'imagine commence à apparaître bien avant le $17^{\mathrm{e}}$ siècle, dans les passages au discours direct le verbe est très rare avant la première tranche : on ne compte que 5 occurrences avant 1610 .

Premièrement, on voit que la construction $[\mathrm{V} \Rightarrow \mathrm{EN}]$ conserve un rôle important dans trois des quatre réseaux. Par exemple, des phrases comme la suivante se trouvent souvent, même au $20^{\mathrm{e}}$ siècle : 
(18) J'imagine très bien un charlatan s'installant dans le Middle West, et pour frapper les imaginations, accréditant cette histoire invérifiable. (Jules Romains, Quand le navire, 1929)

Autrement dit, j'imagine garde son signifié conceptuel cognitif original à côté de son nouveau signifié procédural épistémique.

La construction avec l'infinitif reste secondaire dans les quatre réseaux. La différence de fréquence entre les deux sous-constructions de $[\mathrm{V} \Rightarrow \mathrm{C}]$ qui ressort dans le diagramme 1 est confirmée par les quatre réseaux : la sous-construction [je $[\mathrm{V} \Rightarrow[q u e \Rightarrow \mathrm{C}=\mathrm{ASS}]]]$ prime sur la sous-construction $[j e[\mathrm{~V}=\mathrm{ASS} \Rightarrow[q u e \Rightarrow \mathrm{C}]]]$. C'est-à-dire que $j^{\prime}$ imagine que sert surtout à exprimer la prise en charge réduite, plutôt que l'imagination de quelque chose.

À partir du $19^{\mathrm{e}}$ siècle, $j^{\prime}$ 'imagine que, en tant qu'option préférée pour exprimer la prise en charge réduite, commence à être concurrencée par la construction $[\mathrm{P} \rightarrow \mathrm{EVD}] . \mathrm{Au} 20^{\mathrm{e}}$ siècle, cette dernière prime sur toutes les autres constructions :

(19) - Vous n'allez pas le défendre, j'imagine? (Louis Guilloux, Le sang noir, 1935)

Alors qu'au $19^{\mathrm{e}}$ siècle la construction $[\mathrm{P} \rightarrow-\rightarrow \mathrm{EVD}$ ] est encore relativement variée et comprend trois sous-constructions, au $20^{\mathrm{e}}$ siècle, elle n'en comprend plus qu'une, précisément le verbe et son sujet clitique.

\section{Conclusion}

Les premières occurrences de j'imagine s'étendent sur plusieurs siècles et affichent clairement une séquence de stades successifs. En général, on note que la construction dans laquelle le verbe régit une clause précède la construction dans laquelle le verbe est détaché :

$[\mathrm{V} \Rightarrow \mathrm{C}]>[\mathrm{P} \rightarrow \mathrm{EVD}]$

Parmi les deux sous-constructions principales de $[\mathrm{V} \Rightarrow \mathrm{C}]$, la sous-construction dans laquelle le verbe est au premier plan précède celle dans laquelle la clause est au premier plan. On peut donc établir la séquence suivante :

$[j e[\mathrm{~V}=\mathrm{ASS} \Rightarrow[q u e \Rightarrow \mathrm{C}]]]>[j e[\mathrm{~V} \Rightarrow[q u e \Rightarrow \mathrm{C}=\mathrm{ASS}]]]$

Les trois sous-constructions de $[\mathrm{P} \rightarrow \mathrm{EVD}]$ apparaissent selon l'ordre suivant :

$[\mathrm{P} \rightarrow[$ comme $\Rightarrow[j e \mathrm{~V}]] \leftrightarrow \cdots \mathrm{P}]>[\mathrm{P} \rightarrow[j e[\mathrm{PRO}=\mathrm{V}]] \leftrightarrow \cdots \mathrm{P}]>[\mathrm{P} \rightarrow[j e \mathrm{~V}] \leftrightarrow \cdots \mathrm{P}]$

Autrement dit, la sous-construction détachée avec comme est la première à apparaître. Elle est suivie de la sous-construction détachée avec un pronom, puis, à la fin du $18^{\mathrm{e}}$ siècle, de la sous-construction détachée moderne.

L'analyse des quatre réseaux de constructions successifs apporte des éclaircissements importants concernant l'évolution des constructions après leurs premières occurrences. Jusqu'au $20^{\mathrm{e}}$ siècle, $j^{\prime}$ 'imagine conserve deux signifiés et deux usages : le signifié conceptuel cognitif original 'concevoir l'image d'un être ou d'une chose (sous tel ou tel aspect, dans telle ou telle circonstance)' et son nouveau signifié procédural épistémique 'croire, penser, supposer'. Le signifié conceptuel est surtout transmis par les sous-constructions de [V $\Rightarrow \mathrm{EN}]$ et rarement par la sous-construction $[j e[\mathrm{~V}=\mathrm{ASS} \Rightarrow[q u e \Rightarrow \mathrm{C}]]]$. Le signifié procédural est transmis moyennant les sous-constructions de $[\mathrm{P} \rightarrow \mathrm{EVD}]$ et la sousconstruction [je $[\mathrm{V} \Rightarrow[q u e \Rightarrow \mathrm{C}=\mathrm{ASS}]]$. Dès le premier réseau $\left(17^{\mathrm{e}}\right.$ siècle $)$, le signifié procédural prime sur le signifié conceptuel. On note toutefois que l'expression du signifié procédural change considérablement selon le réseau concerné. Aux $17^{\mathrm{e}}$ et $18^{\mathrm{e}}$ siècles, ce signifié est exprimé surtout par j'imagine que, tandis que la construction détachée joue un 
rôle marginal. Au $19^{\mathrm{e}}$ siècle, la construction détachée commence à gagner de l'importance, au $20^{\mathrm{e}}$ siècle, elle prime sur j'imagine que. Même si la construction détachée commence à s'établir grâce à la sous-construction avec comme et grâce à celle avec un pronom objet indirect clitique, ces deux sous-constructions deviennent marginales au $19^{\mathrm{e}}$ siècle et disparaissent totalement au $20^{\mathrm{e}}$ siècle.

\section{Bibliographie et abréviations}

Albrecht, M. (1964). Die Parenthese in Ovids Metamorphosen und ihre dichterische Funktion. Hildesheim : Georg Olms.

Barðdal, J., S. Gildea (2015). Diachronic construction grammar. Epistemological context, basic assumptions and historical implications. Dans J. Barðdal, E. Smirnova, L. Sommerer, S. Gildea (dirs.). Diachronic construction grammar. Amsterdam : Benjamins, 2-49.

$B F M=$ Laboratoire IHRIM (dir.) (2019). Base de français médiéval. Lyon : ENS de Lyon. <txm.bfmcorpus.org>.

Bolkestein, A. M. (1998a). Modalizing one's message in Latin : 'parenthetical' verba sentienti. Dans Ch. M. Ternes, D. Longrée (dirs.). Actes du colloque Oratio soluta-Oratio numerosa. Les mécanismes linguistiques de cohésion et de rupture dans la prose latine. Bruxelles : Centre Alexandre Wiltheim, 22-33.

Bolkestein, A. M. (1998b). Between brackets : (some properties of) parenthetical clauses in Latin. An investigation of the language of Cicero's letters. Dans R. Risselada (dir.). Latin in use. Amsterdam studies in the pragmatics of Latin. Amsterdam : J. C. Gieben, 1-17.

Buridant, Claude 2019. Grammaire du français médiéval (XIe-XIVe siècles). Strasbourg : Éditions de linguistique et de philologie.

Charnavel, I. (2007). Les énoncés parenthétiques chez Cicéron : syntaxe et pragmatique. Mémoire de Master 2, Université de Paris IV - Sorbonne.

Coleman, K. M. (2010). Parenthetical remarks in the Silvae. Dans E. Dickey, A. Chahoud (dirs.). Colloquial and literary Latin. Cambridge : Cambridge University Press, 292-317.

Féron, C. (2005). Modalisation et verbes d'opinion : quelques remarques sur croire, cuidier et penser dans La Queste del Saint Graal. L'information grammaticale 104, 15-21.

Frantext $=$ ATILF (dir.) (2019). Frantext. Nancy : CNRS - Université de Lorraine. $<$ https://www.frantext.fr>.

Ghezzi, C., P. Molinelli (2014). Deverbal pragmatic markers from Latin to Italian (Lat. quaeso and It. prego) : The cyclic nature of functional developments. Dans C. Ghezzi, P. Molinelli (dirs.). Discourse and pragmatic markers from Latin to the Romance languages. Oxford : Oxford University Press, 61-85.

Glikman, J. (2012). Les incises en croire et cuidier en ancien français. Linx 61, 71-85.

Glikman, J., S. Schneider (2018). Constructions parenthétiques, marques d'oralité et type de textes en diachronie du français. Dans W. Ayres-Bennett, A. Carlier, J. Glikman, T. M. Rainsford, G. Siouffi (dirs.). Nouvelles voies d'accès au changement linguistique. Paris : Classiques Garnier, 317-334.

Haßler, G. (2014). Étude comparée de l'usage parenthétique des verbes épistémiques dans trois langues romanes. Discours 14, <http://discours.revues.org/8888>.

Häusler, S. (2000). Parenthesen im Lateinischen am Beispiel der Pliniusbriefe. Glotta 76, 202-231.

Hilpert, M. (2013). Constructional change in English. Developments in allomorphy, word formation, and syntax. Cambridge : Cambridge University Press. 
Hofmann, J. B. (1951). Lateinische Umgangssprache. 3e édition. Heidelberg : Winter.

Holford-Strevens, L. (2010). Current and ancient colloquial in Gellius. Dans E. Dickey, A. Chahoud (dirs.). Colloquial and literary Latin. Cambridge : Cambridge University Press, 331-338.

Kay, N. M. (2010). Colloquial Latin in Martial's epigrams. Dans E. Dickey, A. Chahoud (dirs.). Colloquial and literary Latin. Cambridge : Cambridge University Press, 318-330.

Lowrey, B. (2018). La constructionalisation et les compléments des verbes de perception. Dans S. Hancil (dir.). Fonctionnements linguistiques et grammaticalisation. Limoges : Lambert-Lucas, 147-171.

Pinkster, H. (1988 [1984]). Lateinische Syntax und Semantik. Tübingen : UTB Francke [Traduction de Latijnse Syntaxis en Semantiek, Amsterdam : Grüner].

Reinhardt, T. (2010). Syntactic colloquialism in Lucretius. Dans E. Dickey, A. Chahoud (dirs.). Colloquial and literary Latin. Cambridge : Cambridge University Press, 203-228.

Risselada, R. (1989). Latin illocutionary parentheticals. Cahiers de l'Institut de Linguistique de Louvain $15,367-378$.

Schneider, S. (2012). Les clauses parenthétiques dans les textes de la Nouvelle-France du $17^{\mathrm{e}}$ et du $18^{\mathrm{e}}$ siècle : aspects pragmatiques, syntaxiques et diachroniques. Linx 61, 87-102.

Schneider, S. (2013). Parenthetische Teilsätze in mittelfranzösischen Texten des 14. und 15. Jahrhunderts. Zeitschrift für romanische Philologie 129, 867-887.

Schneider, S. (2018). Verbos cognitivos en el Corpus del Nuevo diccionario histórico (CDH). RILCE. Revista de filología hispánica 34, 1081-1103.

Schneider, S., J. Glikman (2015). Origin and development of French parenthetical verbs. Dans S. Schneider, J. Glikman, M. Avanzi (dirs.). Parenthetical verbs. Berlin : De Gruyter, 163-188.

Traugott, E. C., G. Trousdale (2013). Constructionalization and constructional changes. Oxford : Oxford University Press. 
${ }^{1}$ Les traductions sont de nous. 